\title{
EL AMPLIFICADOR DE POTENCIA DOHERTY CON ETAPA DE PRE-AMPLIFICACIÓN
}

\author{
(The Doherty Power Amplifier with pre-amplification stage)
}

Jorge Julián Moreno Rubio, Jonathan Javier Tinjacá Soler, William Alexander Cuevas Carrero, Edison Ferney Angarita Malaver

Escuela de Ingeniería Electrónica, Uptc. Grupo Inv. GINTEL, jorgejulian.moreno@uptc.edu.co, jonathan.ts@hotmail. es,wac_401@hotmail.com, edison.angarita@uptc.edu.co

(Recibido 27 de abril 2015 y aceptado 20 de mayo 2015)

\begin{abstract}
Resumen:
Este artículo presenta el concepto general del amplificador de potencia Doherty, resaltando su superioridad, en términos de eficiencia promedio, con respecto a amplificadores de una etapa. Además, se presenta la posibilidad de incluir etapas de pre-amplificación en las ramas del amplificador Doherty, con el objetivo de incrementar su ganancia
\end{abstract}

Palabras clave: Amplificador de potencia, Doherty, comunicaciones inalámbricas, alta ganancia, eficiencia.

\section{INTRODUCCIÓN}

En la etapa de transmisión de un sistema de comunicaciones, existen distintos bloques que ejercen una función específica para llevar a cabo la transferencia de datos (Proakis et al., 1994, Haykin et al., 1989), entre estos se tiene el modulador, el amplificador de potencia y la antena transmisora. En la etapa de amplificación, el amplificador de potencia (PA, del inglés Power Amplifier) es el encargado de incrementar la potencia de las señales para su posterior transmisión, en otras palabras, es el encargado de transformar potencia DC (tomada de una fuente externa) en potencia de radiofrecuencia RF para incrementar la energía de la señal a transmitir (Cripps et al., 2002, Colantonio et al., 2009); en este propósito, el PA debe operar con la máxima eficiencia posible para evitar desperdicio de energía y minimizar los costos de transmisión, siendo este objetivo un campo de investigación vigente por un buen periodo de tiempo (Kim et al., 2010).

\section{Abstract:}

This paper shows the general concept of the Doherty power amplifier, highlighting its superiority, in terms of average efficiency, regarding to single-stage power amplifiers. Furthermore, the posibility of including preamplification stages, in both branches of the Doherty power amplifier is presented, with the goal of increasing its gain.

Key words: Power amplifier, Doherty, wireless communication, high gain, efficiency.

Otra característica de estos sistemas de comunicaciones, es que a mayor frecuencia de operación, mayor es el ancho de banda, permitiendo así transmitir mayor cantidad de información en menor tiempo (Pozar, 1998); pero con el inconveniente de que a más frecuencia surgen algunos inconvenientes como la disminución de la ganancia por parte de los dispositivos amplificadores y además efectos indeseados debido a las redes parásitas que se presentan. Por este motivo, se requiere plantear un modelo de amplificadores con características adecuadas para soportar estas nuevas condiciones.

Algunas topologías de dispositivo único en un PA más utilizadas en radio frecuencia son los amplificadores con sintonización de armónicos (Tuned Load, Class $\mathrm{F}$, entre otros) y arquitecturas más complejas como el Amplificador de Potencia Doherty. En este artículo se presenta una perspectiva general resaltando la importancia de un amplificador de potencia con arquitectura Doherty. Adicionalmente, se muestra en forma general una estrategia usada para aumentar la ganancia en este tipo de 
amplificadores, exponiendo los resultados obtenidos en algunos trabajos que implementan esta estrategia.

\section{IMPORTANCIA DEL AMPLIFICADOR DOHERTY}

El amplificador de potencia Doherty (DPA, del inglés Doherty Power Amplifier) fue propuesto por W. H. Doherty en 1936 (Doherty, 1936). Este inicialmente consistió de dos amplificadores de tubo de vacío y una red inversora de impedancia; con la idea de mejorar la eficiencia, los costos energéticos y reducir el tamaño de los dispositivos con respecto a los amplificadores de potencia lineales como el clase B. En la actualidad, este concepto tan trascendental, ajustado con el desarrollo de nuevos dispositivos de alta movilidad de electrones HEMT combina el manejo de alta potencia, alta frecuencia y alta eficiencia.

Un PA de una sola etapa tiene alcanza su máxima de eficiencia solo en saturación (cripps et al,. 2006, Colantonio 2009), es decir, cuando la amplitud de la señal modulada en la entrada es máxima, lo cual no ocurre de forma constante ya que el tipo de señales usadas en estos sistemas tienen una alta relación de potencia pico a promedio PAPR. Por lo tanto, el PA opera la mayor parte del tiempo con una potencia de salida baja provocando una eficiencia media pobre (Bumman et al,. 2010). En la figura 1, se puede observar un ejemplo de una distribución típica de generación de potencia y la eficiencia como funciones de la potencia de salida en un PA de una sola etapa (Moreno et al,. 2015).

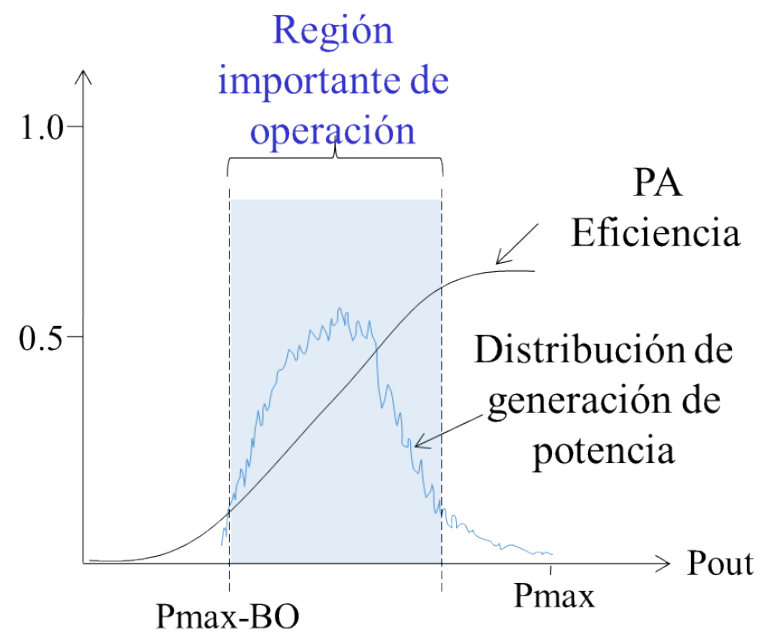

Figura 1. Distribución típica de generación de energía y eficiencia de un PA de una sola etapa Vs. Potencia de salida Pout.

El DPA aparece como una de las herramientas más utilizadas para combatir la baja eficiencia media de un PA de una sola etapa, ya que conserva una eficiencia maximizada en un rango desde bajas hasta altas potencias de salida, como se puede observar en la figura 2.

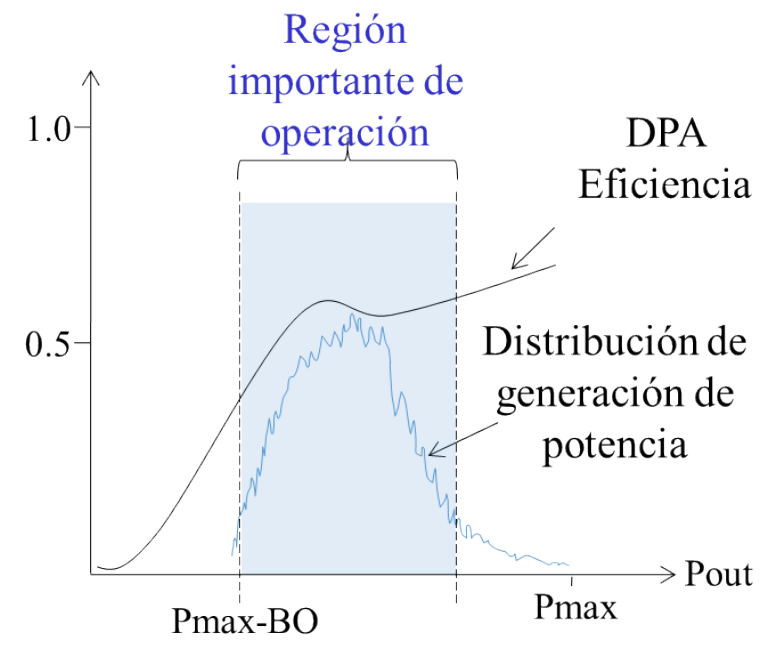

Figura 2. Distribución típica de generación de energía y eficiencia de un DPA.

\subsection{Topología del Amplificador de Potencia Doherty}

El esquema general de un DPA se presenta en la figura 3 , aquí se puede notar que el DPA consta de dos ramas amplificadoras, principal (main) y auxiliar (peak). A la salida del amplificador principal se encuentra e inversor de impedancia y la carga común RL, la cual, es también conectada a la salida del amplificador auxiliar. El divisor de potencia se encarga principalmente de suministrar una porción adecuada de potencia a cada rama amplificadora dependiendo de los requisitos de diseño (Moreno et al,. 2015). 


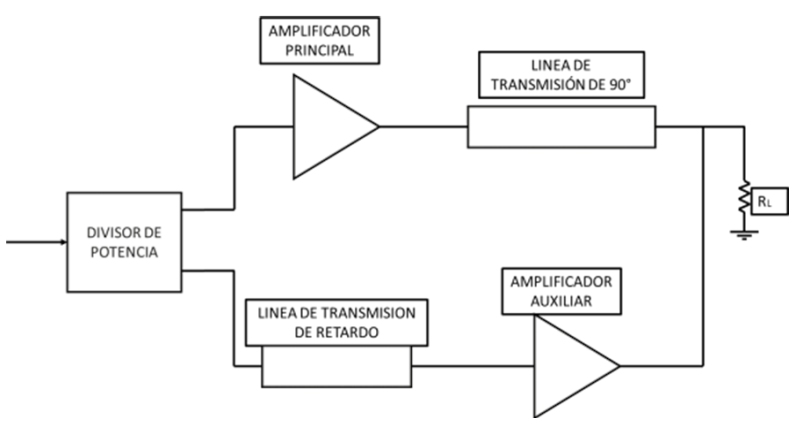

Figura 3. Esquema general de un DPA.

Basado en el comportamiento de la eficiencia en un DPA se pueden resaltar tres importantes regiones: Región de baja potencia, región Doherty y región de saturación.

En la región de baja potencia el amplificador principal se encuentra encendido (polarizado como un clase $\mathrm{AB}$ ) inyectando una corriente a la carga común RL, mientras el amplificador auxiliar está totalmente apagado (polarizado en clase C). Al incrementar la potencia de entrada el amplificador principal alcanzará su máxima eficiencia de drenaje siendo hasta el momento también la eficiencia total del DPA. Este punto es conocido como Break Point.

Si la potencia de entrada sigue incrementando el amplificador auxiliar se enciende. Por lo tanto, se administra una corriente a la carga común RL proveniente del amplificador auxiliar causando que la eficiencia del DPA esté dada por la eficiencia ponderada de los amplificadores de las ramas principal y auxiliar. Por último, si sigue incrementando la potencia de entrada se presentaría la completa saturación del DPA. En la figura 4 se muestra el perfil típico de la eficiencia Vs la Potencia de salida en un DPA.

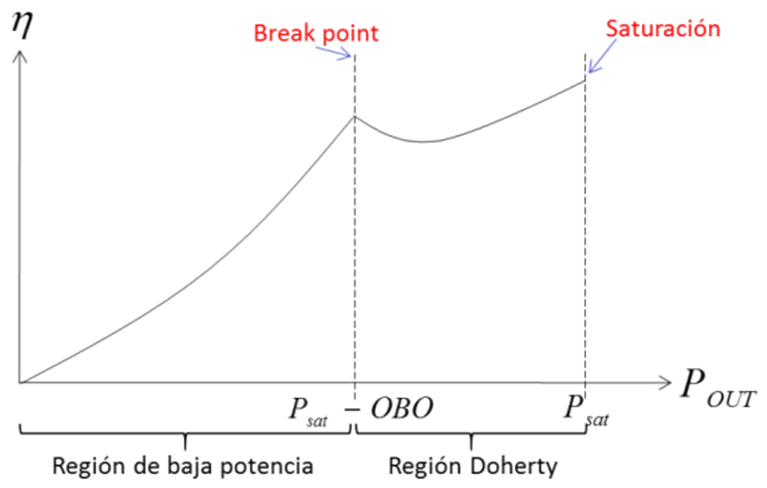

Figura 4. Curva de eficiencia típica en un DPA.

Como se puede apreciar en la figura 4, un parámetro importante en un DPA, es la potencia de salida en la cual se presenta el primer pico de eficiencia del DPA (Break point), ya que, la diferencia entre la potencia de salida en saturación y la potencia de salida en el Break Point define la región Doherty. Es decir, la región en la cual la eficiencia del DPA es maximizada. Esta diferencia en dBs es conocida como output-back-off (OBO), el cual se puede interpretar como el tamaño en $\mathrm{dBs}$ de la región Doherty.

En la tabla 1, se presentan los resultados de algunos diseños de DPA donde se especifica su desempeño en cuanto a la frecuencia de trabajo, eficiencia, potencia de salida máxima y ganancia.

Tabla 1: Resultados importantes de algunos DPAs desarrollados.

\begin{tabular}{cccccc}
\hline DPA & Frecuencia & Ganancia & $\mathbf{P}_{\text {out }}$ & \multicolumn{2}{c}{$\begin{array}{c}\text { Eficiencia \% } \\
\text { @ 6dB }\end{array}$} \\
& $\mathbf{G H z}$ & $\mathbf{d B}$ & $\mathbf{d B m}$ & $\begin{array}{l}\text { OBO } \\
\text { OBO }\end{array}$ \\
\hline Moreno2011 & 3.5 & 8 & 43.5 & 57 & 65 \\
Markoz2010 & 5.6 & 8 & 34 & 26 & 40.5 \\
Gruner 2011 & 6 & 12 & 41.5 & 49 & 63 \\
Chen 2015 & 2.3 & 14 & 40.5 & 40.5 & 43 \\
Moreno2012 & 3.5 & 8.5 & 44 & 50 & 60 \\
Moreno2012 & 3.5 & 10 & 43.2 & 55 & 61 \\
\hline
\end{tabular}

En estos trabajos normalmente la ganancia decae en la región Doherty. Por lo tanto, es necesario desarrollar estrategias para aumentar la ganancia en un DPA. Una de ellas es la pre amplificación de las ramas principal y/o auxiliar.

\section{ESTRATEGIA PARA INCREMENTAR LA GANANCIA DE UN DPA}

En la figura 5, se presenta la estructura de un DPA pre amplificando sus ramas principal y auxiliar. 


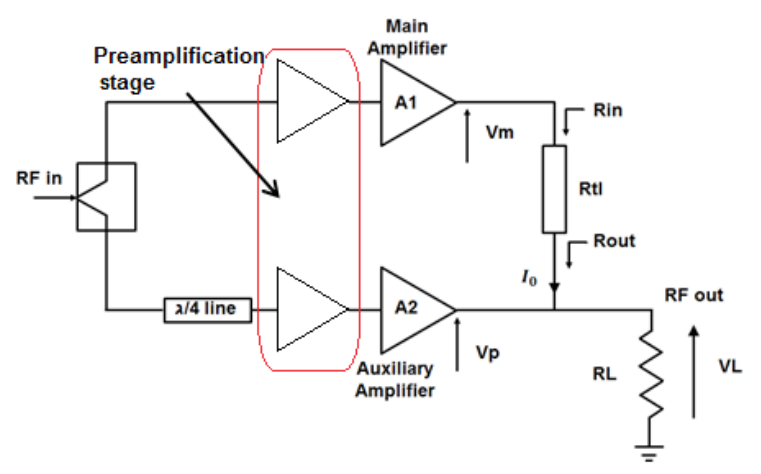

Figura 4. DPA con etapa de pre amplificación.

Con una pre-amplificación clase $\mathrm{AB}$ en las ramas principal y/o auxiliar aumenta la ganancia de los amplificadores. Por lo tanto, se incrementa la ganancia total del DPA. Sin embargo, la eficiencia se ve reducida debido al consumo de energía al agregar más dispositivos.

En la tabla 2 se muestran los resultados obtenidos en diferentes trabajos que modifican la arquitectura convencional de un DPA pre-amplificando sus ramas principal y/o auxiliar. Podemos notar que efectivamente la eficiencia es reducida. Sin embargo la ganancia es incrementada significativamente comparado con los resultados de los trabajos de la tabla1.

Tabla 2: Resultados importantes de algunos DPAs con etapas de pre amplificación.

\begin{tabular}{cccccc}
\hline DPA & $\begin{array}{c}\text { Frecuencia } \\
\text { GHz }\end{array}$ & $\begin{array}{c}\text { Ganancia } \\
\mathbf{d B}\end{array}$ & $\begin{array}{c}\mathbf{P}_{\text {OUT }} \\
\mathbf{d B m}\end{array}$ & $\begin{array}{c}\text { EdB } \\
\mathbf{6 d B}\end{array}$ & $\begin{array}{c}\text { OdB } \\
\text { OBO }\end{array}$ \\
\hline Ghim 2006 & 2.1 & 27 & 40 & 25 & 27 \\
Crescenzi2005 & 2 & 26 & 47.8 & 35 & 40 \\
Chang 2015 & 2.6 & 27 & 45 & 42 & 55 \\
Lee 2014 & 2.1 & 10.9 & 51.4 & 55 & 58 \\
Park 2015 & 1.9 & 13 & 44.3 & 57.2 & 60.5 \\
\hline
\end{tabular}

\section{CONCLUSIONES}

Se presenta el panorama de un DPA con el fin de apreciar su importancia debido a sus buenos resultados en comparación con otro tipo de estructuras en términos de eficiencia. Adicionalmente se muestra una estrategia para el diseño de amplificadores de potencia en base a la topología propuesta por Doherty, con el fin de obtener un incremento en la ganancia para altas frecuencias de trabajo.

\section{REFERENCIAS}

Proakis, J., Salehi, M., Zhou, N. \& Li, X. 1994., Communication systems engineering, Prentice-Hall.

Haykin, S., Moher, M. \& Song, T. 1989. An introduction to analog and digital communications, Wiley.

Cripps, S. 2002. Advanced techniques in RF power amplifier design. Artech House on Demand.

Colantonio, P., Giannini, F. \& Limiti, E. 2009. High Efficiency $R F$ and Microwave Solid State Power Amplifiers, Wiley Online Library. doi: 10.1002/9780470746547.

Kim, B., Moon, J. \& Kim, I. 2010. Efficiently amplified. IEEE Microwave Magazine. 11(5). pp. 87-100. doi: 10.1109/MMM.2010.937099.

Pozar, D. M. 1998. Microwave Engineering, 2nd. Wiley.

Cripps, S. C. 2006. RF power amplifier for wireless communication, 2nd. Retch House, Incorporated.

Moreno J., Fernandez H., Angarita E. 2015. A $25 \mathrm{~W}$ 70\% Efficiency Doherty Power Amplifier With 6dB Output Back-Off for $2.4 \mathrm{GHz}$ Applications, with VGS,PEAK Control. INGE CUC 11(1). pp. 48-52. doi:http://dx.doi.org/10.17981/ingecuc.11.1.2015.04

Moreno J., Angarita E. 2015. The Doherty Power Amplifier: Design Strategy. Proceedings of the 2015 IEEE Thirty Fifth Central American and Panama Convention (CONCAPAN XXXV).

Moreno J., Fang J., Camarchia V., Quaglia R., Pirola M., Ghione G., S. D. Guerrieri. 2011. A $22 \mathrm{~W}$ 65\% efficiency GaN Doherty Power Amplifier at $3.5 \mathrm{GHz}$ for WiMAX applications. Workshop on Integrated Nonlinear Microwave and Millimetre-Wave Circuits (INMMIC). doi: 10.1109/ INMMIC.2011.5773332. 
A. Z. Markos*, D. Gruner, K. Bathich, \& G. Boeck. 2010. A 2 W GaAs doherty amplifier for 5.5-5.6 $\mathrm{GHz}$ applications. 18th International Conference on Microwave Radar and Wireless Communications (MIKON).

Gruner D., Bathich K., Tanany A., \& Boeck G. 2011. Harmonically tuned GaN-HEMT Doherty power amplifier for $6 \mathrm{GHz}$ applications. Microwave Integrated Circuits Conference (EuMIC), 2011.

Chen W., Jia S., Schreurs D. 2015. High efficiency Doherty transmitter with antenna active load modulation. Wireless Symposium (IWS), 2015 IEEE International. doi: 10.1109/IEEEIWS.2015.7164510

Rubio, J. M., Fang, J., Camarchia, V., Quaglia, R., Pirola, M. \& Ghione, G. 2012. 3-3.6-GHz wideband $\mathrm{GaN}$ Doherty power amplifier exploiting output compensation stages. Microwave Theory and Techniques, IEEE Transactions on, 60, 25432548.

Fang, J., Moreno, J., Quaglia, R., Camarchia, V., Pirola, M., Guerrieri, S. D., Ramella, C. \& Ghione, G. 2012. 3.5 GHz WiMAX GaN Doherty power amplifier with second harmonic tuning. Microwave and Optical Technology Letters, 54(11). pp. 2601-2605. doi: 10.1002/mop.
Ghim J., Cho K., Kim J., Stapleton S. 2006. A High Gain Doherty Amplifier Using Embedded Drivers. IEEE MTT-S International Microwave Symposium Digest. doi: 10.1109/ MWSYM.2006.249753.

Crescenzi E., Pengelly R., Wood S., Buss R. 2005. 60 Watt Doherty Amplifiers Using High Gain 2-Stage Hybrid Amplifier ModuleS. IEEE MTT-S International Microwave Symposium Digest. doi: 10.1109/MWSYM.2005.1516941.

Chang D., Noh Y., Yom I. 2015. GaN High Power Amplifier MMIC for $30 \mathrm{~W}$ Compact Doherty Amplifier for LTE Active Antenna System. Proceedings of the 10th European Microwave Integrated Circuits Conference. pp. 265-268. doi: 10.1109/EuMIC.2015.7345120.

Lee J., Son J., Kim N., 2014. Optimised Doherty power amplifier with auxiliary peaking cell. Electronics Letters 50(18). pp. 1299-1301. doi: 10.1049/el.2014.2214.

Park Y., Lee J., Jee S., Kim S., Kim B. 2015. Gate Bias Adaptation of Doherty Power Amplifier for High Efficiency and High Power. IEEE Microwave and Wireless Components Letters 25(2). PP. 136-138. doi: 10.1109/LMWC.2014.2373637. 